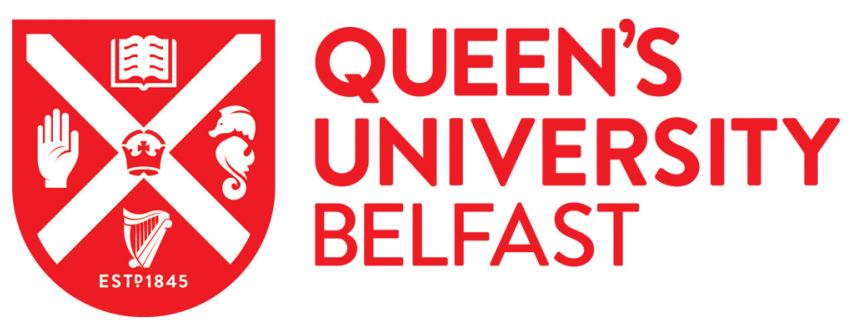

\title{
Trends in new prescription of gabapentinoids and of coprescription with opioids in the 4 nations of the UK, 1993-2017
}

Rahman, A., Kane, J., Montastruc, F., \& Renoux, C. (2021). Trends in new prescription of gabapentinoids and of coprescription with opioids in the 4 nations of the UK, 1993-2017. British Journal of Clinical Pharmacology, 87(8), 3349-3353. https://doi.org/10.1111/bcp.14727

Published in:

British Journal of Clinical Pharmacology

Document Version:

Peer reviewed version

Queen's University Belfast - Research Portal:

Link to publication record in Queen's University Belfast Research Portal

Publisher rights

Copyright 2021 Wiley. This work is made available online in accordance with the publisher's policies. Please refer to any applicable terms of use of the publisher.

\section{General rights}

Copyright for the publications made accessible via the Queen's University Belfast Research Portal is retained by the author(s) and / or other copyright owners and it is a condition of accessing these publications that users recognise and abide by the legal requirements associated with these rights.

Take down policy

The Research Portal is Queen's institutional repository that provides access to Queen's research output. Every effort has been made to ensure that content in the Research Portal does not infringe any person's rights, or applicable UK laws. If you discover content in the Research Portal that you believe breaches copyright or violates any law, please contact openaccess@qub.ac.uk. 
Renoux Christel (Orcid ID: 0000-0002-4691-9579)

\section{Trends in new prescription of gabapentinoids and of coprescription with opioids in the four nations of the United Kingdom, 1993-2017}

Alvi Rahman, MSc ${ }^{1,2}$; Joseph Kane, MB BaO BCh, MRCPsych, $\mathrm{PhD}^{3}$; François Montastruc, $\mathrm{MD}, \mathrm{PhD}^{4,5}$; Christel Renoux, MD, $\mathrm{PhD}^{2,6}$

${ }^{1}$ Department of Epidemiology, Biostatistics and Occupational Health, McGill University, Montreal,

Canada

${ }^{2}$ Centre for Clinical Epidemiology, Lady Davis Institute for Medical Research, Jewish General

Hospital, Montreal, Canada

${ }^{3}$ Centre for Public Health, Queen's University Belfast, Belfast, Northern Ireland, United Kingdom

${ }^{4}$ Department of Medical and Clinical Pharmacology, Centre of PharmacoVigilance and

Pharmacoepidemiology, Toulouse University Hospital, Faculty of Medicine, Toulouse, France

${ }^{5}$ INSERM, UMR 1027 Pharmacoepidemiology, Assessment of Drug Utilization and Drug Safety, CIC 1426 - University Paul Sabatier Toulouse, Toulouse, France

${ }^{6}$ Department of Neurology and Neurosurgery, McGill University, Montreal, Canada

\section{Corresponding Author:}

Christel Renoux, MD, PhD

Centre for Clinical Epidemiology, Lady Davis Institute, Jewish General Hospital

3755 Côte Sainte Catherine H 416.1

Montréal, QC

H3T 1E2, Canada

Telephone: (514) 340-7563

Fax: (514) 340-7564

Email: christel.renoux@mcgill.ca

This article has been accepted for publication and undergone full peer review but has not been through the copyediting, typesetting, pagination and proofreading process which may lead to differences between this version and the Version of Record. Please cite this article as doi: $10.1111 /$ bcp. 14727 
Manuscript word count: 1731

Abstract word count: 144

Keywords: gabapentin, pregabalin, gabapentinoid, opioids, drug utilization

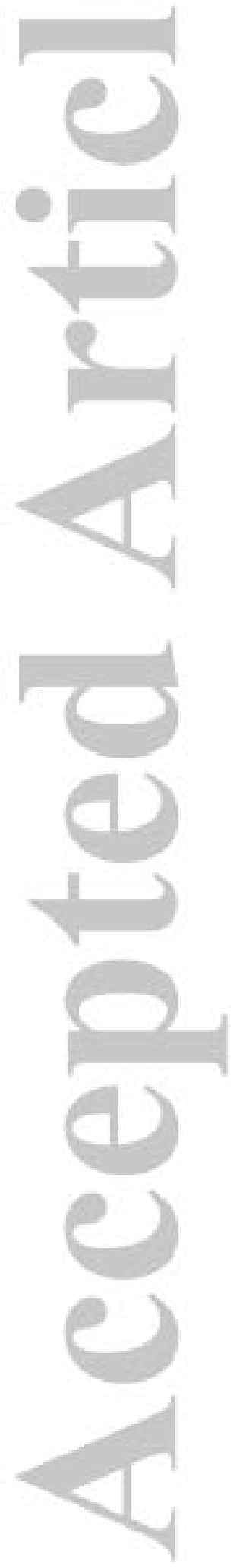

This article is protected by copyright. All rights reserved. 


\section{WHAT IS ALREADY KNOWN ABOUT THIS SUBJECT}

- Gabapentinoid drugs, which include gabapentin and pregabalin, are prescribed in the United Kingdom for the treatment of epilepsy, neuropathic pain, and generalized anxiety disorders (pregabalin).

- Gabapentinoid drugs cause depression of the central nervous system and have the potential for physical dependency and abuse.

- In the last decade, there has been a tripling of gabapentinoid prescriptions in the United Kingdom.

\section{WHAT THIS STUDY ADDS}

- The rate of new gabapentin prescriptions in Northern Ireland rapidly increased after 2010 and exceeded that of the other nations by 2017.

- The annual rate of new pregabalin prescriptions was constantly higher in Northern Ireland than in the other UK nations, up to five times greater in 2010.

- The proportion of patients prescribed gabapentinoids who were coprescribed opioids was approximately 15 to $20 \%$ in each UK nation during the study period. 


\section{ABSTRACT}

We explored potential differences in time trends of gabapentinoid prescription and of opioid coprescription between 1993 and 2017 in the four UK nations using the Clinical

Practice Research Datalink, a UK primary care database. There were distinct trends in annual rates of new gabapentin and pregabalin prescriptions in Northern Ireland. The rate of new gabapentin prescriptions rapidly increased after 2010 and exceeded that of the other nations by 2017 (rate of 836 (95\% CI: 787-887) per 100,000 person-years). Additionally, the rate of new pregabalin prescriptions was higher during the entire study period, reaching a peak of 1,139 (95\% CI: $1,088-1,193)$ per 100,000 person-years in 2010, five-fold higher than the other nations. Findings in Northern Ireland may be partly attributable to the high burden of anxiety disorders, an indication for pregabalin. Further exploration of reasons for discrepancies in gabapentinoid prescribing between UK nations is warranted. 


\section{INTRODUCTION}

Gabapentinoid drugs, namely gabapentin and pregabalin, were approved in the United Kingdom (UK) in 1993 and 2004, respectively, for the treatment of epilepsy, neuropathic pain, and generalized anxiety disorder (pregabalin). Several reports implicate gabapentinoids with physical dependency, potential for abuse, and central nervous system (CNS) depression, the risk of which may be augmented by concomitant use of opioids.[1,2] Evidence from randomized trials shows that gabapentin is associated with respiratory depression compared with placebo.[3,4] The concomitant use of gabapentinoids and opioids may increase the risk of opioid-related respiratory depression and overdose. In a randomized crossover study, pregabalin potentiated the respiratory depressant effect of remifentanil, a potent short-acting opioid, by 62\%.[5] Moreover, a nested case-control study of adults aged 65 years and above registered in the Ontario Drug Benefit Plan reported that opioid users with concomitant use of gabapentin had a $49 \%$ increased risk of opioid-related death compared to opioid use alone.[6] Given these potential risks, monitoring the prescribing of gabapentinoids is essential.

Over the last decade, there has been a tripling of gabapentinoid prescriptions in the UK and United States[7,8] and it has been hypothesized that this rise may be partly explained by the off-label prescribing for the management of any type of pain. [9] However, it is unknown whether the rates of gabapentinoid prescriptions as well as of opioid coprescriptions vary across the four UK nations. Bridging this knowledge gap may have practice and policy implications regarding the prescribing of these drugs. Therefore, we estimated the annual rate of patients newly prescribed gabapentin and pregabalin in each UK nation (England,

Northern Ireland, Scotland, and Wales) and the proportion of these patients concomitantly prescribed opioids.

\section{METHODS}


We used the Clinical Practice Research Datalink (CPRD), a UK primary care electronic medical records database of over 15 million patients from more than 700 practices, which is representative of the general population according to age, sex, and ethnicity.[10] Of all practices included, $76 \%$ were in England, $11 \%$ in Scotland, 10\% in Wales, and 3\% in Northern Ireland. The CRPD records information on demographics, lifestyle (e.g. smoking and alcohol consumption), prescriptions, medical diagnoses, and referrals. Information pertaining to drug prescriptions are recorded automatically at the time of issue by the general practitioner. Quality control audits are conducted regularly to ensure accuracy and completeness of data.[10]

We formed a cohort of patients registered for at least one day between January 1, 1993 and December 31, 2017. We defined the start of follow-up as January 1, 1993, a patient's registration date with a practice, or the date the practice started contributing valid data, whichever occurred later. Follow-up ended on the date that the patient transferred out of the practice, the date of death, or December 31, 2017, whichever occurred first. To identify new users of gabapentin and pregabalin, we excluded patients with a prescription for these drugs any time before cohort entry. We estimated separately the crude annual rates and $95 \%$ confidence intervals (CI), per 100,000 person-years (PY), of patients newly prescribed gabapentin and pregabalin in the four UK nations, respectively. We also fitted Poisson regression models to estimate the rate ratio (RR) between 2007 and 2017 in each nation, adjusted for age and sex. In addition, we estimated a dispersion parameter to adjust for overdispersion [11]. In secondary analyses, we estimated the crude annual rates and 95\% CIs of patients newly prescribed gabapentin and pregabalin with a same-day coprescription for an opioid drug in each nation. Subsequently, we determined the proportion of new gabapentin and pregabalin users coprescribed an opioid drug. 
The study protocol was approved by the Independent Scientific Advisory Committee of the CPRD (No.18_033) and the research ethics board of the Jewish General Hospital in Montreal, Canada, who waived the requirement for informed consent.

Principal Investigator Statement: The authors confirm that the Principal Investigator for this paper is Dr. Christel Renoux.

\section{RESULTS}

Between 1993 and 2017, 12,512,468 patients entered the study cohort. During this period, 256,410 patients $(2.0 \%)$ were newly prescribed gabapentin, of whom $67 \%$ were in England, 15\% in each of Scotland and Wales, and 3\% in Northern Ireland. Between 2004 and 2017, $136,653(1.1 \%)$ patients were newly prescribed pregabalin, of whom $64 \%$ were in England, $13 \%$ in Wales, $12 \%$ in Scotland, and $11 \%$ in Northern Ireland.

In England, Scotland, and Wales, the annual rates of patients newly prescribed gabapentin gradually increased for most of the study period and plateaued from 2015 onwards (Figure 1A). Between 2007 and 2017, the rates increased from 212 to 617 per 100,000 PY (RR: 3.35; 95\% CI: 3.33-3.37) in England, from 369 to 742 per 100,000 PY (RR: 2.25; 95\% CI: 2.212.28) in Scotland, and from 268 to 728 per 100,000 PY (RR: 2.97 ; 95\% CI: $2.93-3.01$ ) in Wales. In Northern Ireland however, following an initial increase, the rate slightly declined in 2004 and stabilized from 2005 to 2009 . Subsequently, the rate sharply increased and surpassed the other nations from 2016 onwards. Between 2007 and 2017, the rate of new gabapentin prescriptions in Northern Ireland increased from 139 to 836 per 100,000 PY (RR: 6.71; 95\% CI: 6.51-6.93). Despite the differences in annual rates between some nations, the rate of concomitant opioid prescriptions changed proportionally with the overall rate of new gabapentin prescriptions, while the annual proportion of concomitant users ranged from 15 to 20\% between 2007 and 2017 in each nation (Figure 2A). 
During the study period, there was a steady increase in the annual rate of patients newly prescribed pregabalin in England, Scotland, and Wales (Figure 1B). Between 2007 and 2017, the rate rose from 118 to 351 per 100,000 PY (RR: 3.37; 95\% CI: 3.35-3.39) in England, from 96 to 418 per 100,000 PY (RR: 4.77; 95\% CI: 4.69-4.85) in Scotland, and from 104 to 370 per 100,000 PY (RR: 3.81; 95\% CI: 3.75-3.87) in Wales. A rapid escalation in the annual rate of first pregabalin prescription was observed in Northern Ireland, from 546 (95\% CI: 511-582) per 100,000 PY in 2007 to 1,139 (95\% CI: 1,088-1,193) per 100,000 PY in 2010, five times higher than the other nations. This peak was followed by a decline to 532 (95\% CI: 493-573) per 100,000 PY in 2017, but the rate remained higher than in the other nations. The rate of concomitant opioid prescriptions changed proportionally with the rate of overall new pregabalin prescriptions (Figure 2B), corresponding to $15-20 \%$ of new pregabalin users concomitantly prescribed opioids between 2007 and 2017 in each nation.

\section{DISCUSSION}

Since their approval, the rates of both new gabapentin and pregabalin prescriptions have increased in all UK nations. However, the trend was distinct in Northern Ireland, with a rapid rise in new gabapentin prescriptions between 2010 and 2017 and notably high rates of new pregabalin prescriptions following its approval. Following its peak in 2010, the decline in the annual rate of new pregabalin prescriptions in Northern Ireland coincided with a rapid rise in new gabapentin prescriptions, suggesting possible replacement of pregabalin with gabapentin. The proportion of patients with a coprescription for opioids remained constant over time and similar between each nation.

Reasons for the notable differences in the trends of gabapentinoid prescriptions between Northern Ireland and the other UK nations are unclear and may be multifactorial. In particular, variation in the prevalence of comorbidities may play a role. With $25 \%$ greater 
psychiatric morbidity than the rest of the UK, Northern Ireland has the highest prevalence of mental health disorders among the UK nations, which is described as a consequence of the 30-year civil conflict known as The Troubles.[12] Among these, anxiety disorders are the most commonly reported.[12,13] The National Institute for Health and Care in Excellence, which provides authoritative guidance on specific conditions to clinical practice in England, Northern Ireland, and Wales, recommended pregabalin for the management of generalized anxiety disorder in 2011. Although we reported an increase in the rate of pregabalin prescribing prior to this recommendation, by 2007, numerous trials had suggested its efficacy in treating generalized anxiety disorder.[14-17] Furthermore, the proportion of the health budget in Northern Ireland devoted to the provision of mental health is $6 \%$, which is half that of England.[12] Hence, potential underfunding may have led to inadequate availability of psychological and mental health services, resulting in an increase of pharmacological therapy, including the use of pregabalin.[12] Another contributing factor may be the rise of gabapentinoid abuse in Northern Ireland, which caused a greater number of drug-related deaths than individual opioids in 2017 and 2018.[18]. This rise may be attributed to the increased prescribing of gabapentinoids as an alternative to opioids for the management of any type of pain.[19] The risk of gabapentinoid misuse, if administered at therapeutic dosages, may be lower than that of drugs such as benzodiazepines and opioids, thus some may perceive gabapentinoids as a valid substitute.[20] However, reports of abuse and misuse of prescription gabapentin and pregabalin have rapidly increased in recent years, particularly in individuals with a history of substance abuse.[20] Indeed, patients receiving supratherapeutic doses of both gabapentin and pregabalin have reported to experience euphoria, producing effects such as sedation, dissociation, numbness, uninhibited behaviour, or hallucinations.[20-22] Finally, the role of reimbursement practices in explaining the reported differences may also be considered. Albeit, the cost of prescribed drugs is the same 
in all UK nations and there are no major differences in subsidization. In Northern Ireland, Scotland, and Wales, all prescriptions have been free for patients since 2010, 2011, and 2007, respectively, with a cost $£ 3$ prior to these dates. In England, patients currently pay $£ 9.15$ per prescription, irrespective of the drug.[23,24]

The study has some limitations. First, the data do not capture gabapentinoid prescriptions issued by specialists including neurologists and psychiatrists. Nonetheless, general practitioners being the gatekeepers of the UK healthcare, prescriptions issued by specialists are usually renewed by general practitioners, thus the findings reported may still accurately reflect gabapentinoid prescribing trends across UK nations. Second, we were unable to examine indications for new prescriptions of gabapentin and pregabalin and their role in explaining the reported findings as diagnoses in the CPRD are not linked with issued prescriptions.

Given its potential for abuse and risk of respiratory depression in combination with opioids, monitoring the escalating rates of new gabapentinoid prescriptions in the UK nations, particularly in Northern Ireland, is critical. To facilitate the development of interventions, future studies should explore factors that may explain the variation in gabapentinoid prescribing across the nations. 
Acknowledgements: We thank Mrs. Clare Gallagher for her guidance regarding licensing and reimbursement of gabapentinoids across the UK. Mr. Rahman is the recipient of a Canada Graduate Scholarship - Doctoral from the Canadian Institutes of Health Research and the Richard H. Tomlinson Doctoral Fellowship from McGill University. Dr. Renoux is a recipient of a Chercheur-Boursier Salary Award from the Fonds de recherche du Québec Santé. The funders do not have a role in any aspect of the study.

Author Contributions: All authors were involved in study design and conceptualization. AR and CR acquired, analyzed, and interpreted the data and also drafted the manuscript. All authors were involved critical revision of the manuscript for important intellectual content. CR provided study supervision.

Conflicts of Interest: None to declare.

Data Availability: Data cannot be shared due to legal and ethical reasons.

Nomenclature of Targets and Ligands: Key protein targets and ligands in this article are hyperlinked to corresponding entries in http://www.guidetopharmacology.org, and are permanently archived in the Concise Guide to PHARMACOLOGY 2019/20.[25] 


\section{REFERENCES}

1. Fuzier R, Serres I, Guitton E, Lapeyre-Mestre M, Montastruc JL, French Network of Pharmacovigilance $C$. Adverse drug reactions to gabapentin and pregabalin: a review of the French pharmacovigilance database. Drug Saf. 2013;36(1):55-62.

2. Schwan S, Sundstrom A, Stjernberg E, Hallberg E, Hallberg P. A signal for an abuse liability for pregabalin--results from the Swedish spontaneous adverse drug reaction reporting system. Eur J Clin Pharmacol. 2010;66(9):947-953.

3. Piovezan RD, Kase C, Moizinho R, Tufik S, Poyares D. Gabapentin acutely increases the apnea-hypopnea index in older men: data from a randomized, double-blind, placebocontrolled study. J Sleep Res. 2017;26(2):166-170.

4. Cavalcante AN, Sprung J, Schroeder DR, Weingarten TN. Multimodal Analgesic Therapy With Gabapentin and Its Association With Postoperative Respiratory Depression. Anesth Analg. 2017;125(1):141-146.

5. Myhre M, Diep LM, Stubhaug A. Pregabalin Has Analgesic, Ventilatory, and Cognitive Effects in Combination with Remifentanil. Anesthesiology. 2016;124(1):141-149.

6. Gomes T, Juurlink DN, Antoniou T, Mamdani MM, Paterson JM, van den Brink W. Gabapentin, opioids, and the risk of opioid-related death: A population-based nested casecontrol study. PLoS medicine. 2017;14(10):e1002396.

7. Montastruc F, Loo SY, Renoux C. Trends in first gabapentin and pregabalin prescriptions in primary care in the United Kingdom, 1993-2017. JAMA. 2018;320(20):2149-2150.

8. Johansen ME. Gabapentinoid use in the United States 2002 through 2015. JAMA Intern Med. 2018;178(2):292-294.

9. Goodman CW, Brett AS. Gabapentin and Pregabalin for Pain - Is Increased Prescribing a Cause for Concern? N Engl J Med. 2017;377(5):411-414.

10. Herrett E, Gallagher AM, Bhaskaran K, et al. Data Resource Profile: Clinical Practice Research Datalink (CPRD). Int J Epidemiol. 2015;44(3):827-836.

11. McCullagh P, Nelder JA. Generalized Linear Models: Second Edition. Chapman and Hall; 1989.

12. O'Neill S, Rooney N. Mental health in Northern Ireland: an urgent situation. The Lancet Psychiatry. 2018;5(12):965-966.

13. Bunting BP, Murphy SD, O'Neill SM, Ferry FR. Lifetime prevalence of mental health disorders and delay in treatment following initial onset: evidence from the Northern Ireland Study of Health and Stress. Psychol Med. 2012;42(8):1727-1739.

14. Pande AC, Crockatt JG, Feltner DE, et al. Pregabalin in generalized anxiety disorder: a placebo-controlled trial. Am J Psychiatry. 2003;160:533-540.

15. Feltner DE, Crockatt JG, Dubovsky SJ, et al. A randomized, double-blind, placebo-controlled, fixed-dose, multicenter study of pregabalin in patients with generalized anxiety disorder. $J$ Clin Psychopharmacol. 2003;23:240-249.

16. Rickels $\mathrm{K}$, Pollack $\mathrm{MH}$, Feltner $\mathrm{DE}$, et al. Pregabalin for treatment of generalized anxiety disorder. Arch Gen Psychiatry. 2005;62:1022-1030.

17. Baldwin DS, Ajel K. Role of pregabalin in the treatment of generalized anxiety disorder. Neuropsychiatr Dis Treat. 2007;3(2):185-191.

18. Drug-related and drug-misuse deaths 2008-2018. Belfast, Northern Ireland, UK: Northern Ireland Statistics and Research Agency;2020.

19. Peckham AM, Evoy KE, Ochs L, Covvey JR. Gabapentin for Off-Label Use: Evidence-Based or Cause for Concern? Subst Abuse. 2018;12:1178221818801311.

20. Schifano F. Misuse and abuse of pregabalin and gabapentin: cause for concern? CNS Drugs. 2014;28(6):491-496. 
21. Bonnet U, Richter EL, Isbruch K, Scherbaum N. On the addictive power of gabapentinoids: a mini-review. Psychiatr Danub. 2018;30(2):142-149.

22. Bonnet $U$, Scherbaum N. How addictive are gabapentin and pregabalin? A systematic review. Eur Neuropsychopharmacol. 2017;27(12):1185-1215.

23. Help with NHS prescription costs. NHS Business Services Authority

https://www.nhsbsa.nhs.uk/help-nhs-prescription-costs. Accessed November 5, 2020.

24. Prescriptions. NIDirect Government Services. https://www.nidirect.gov.uk/articles/prescriptions. Accessed November 5, 2020.

25. Alexander SPH, Kelly E, Mathie A, et al. The Concise Guide to PHARMACOLOGY 2019/20. Br J Pharmacol. 2019;176:S1-S493.

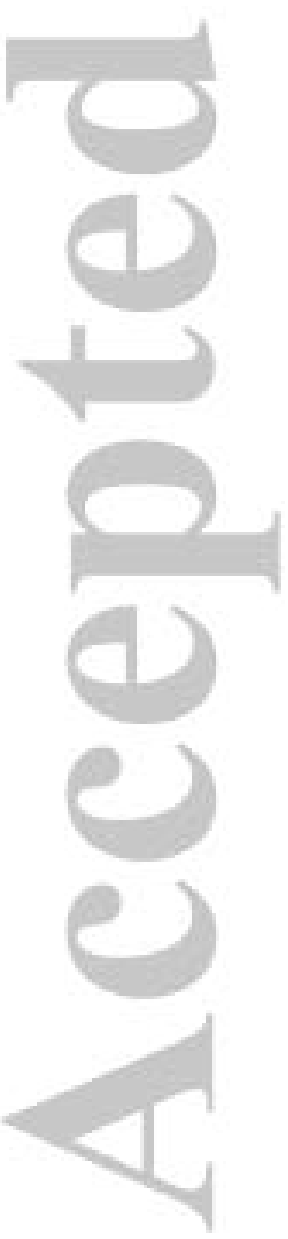

This article is protected by copyright. All rights reserved. 
A Gabapentin

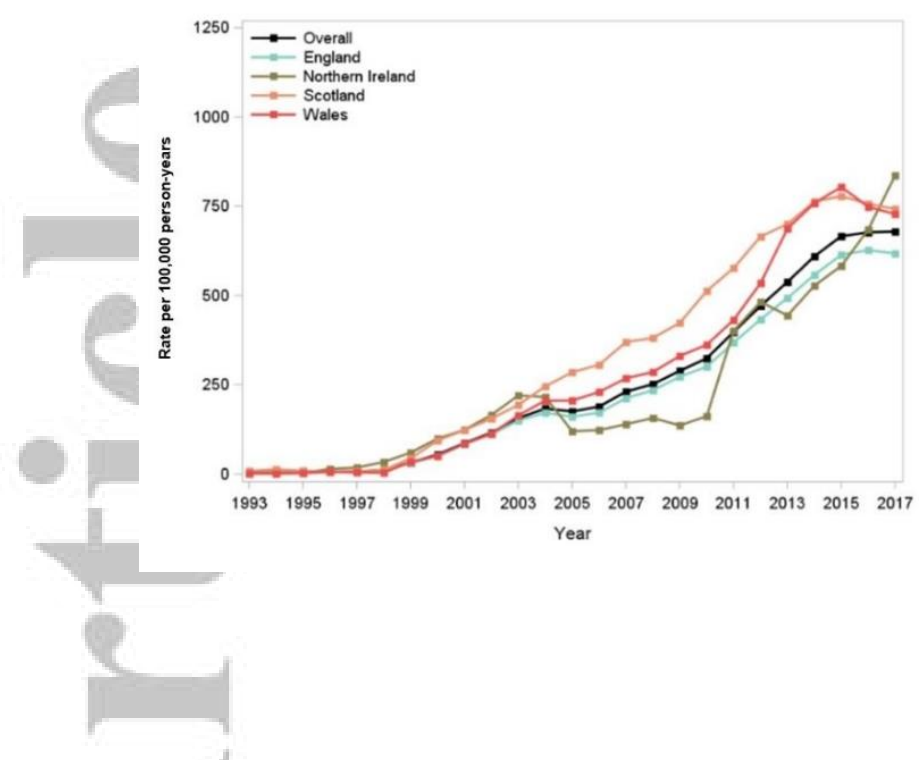

B Pregabalin

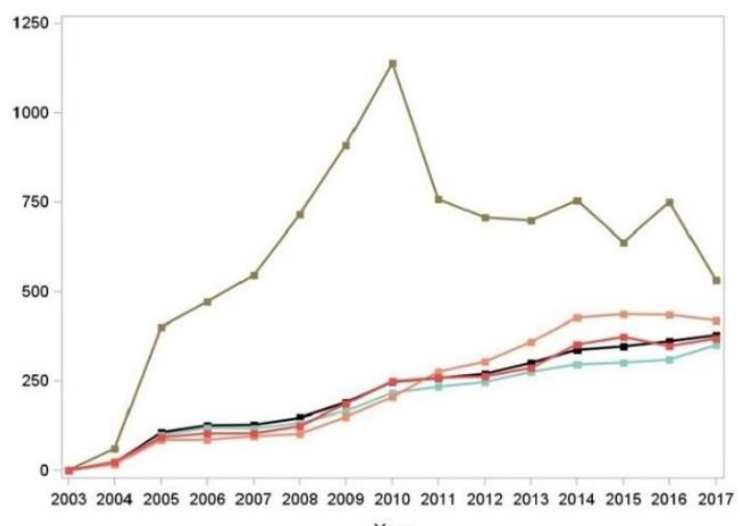

Year

Figure 1. Rates of new users of gabapentin (A) and pregabalin (B) in each UK nation from market approval to 2017. Gabapentin and pregabalin were approved for use in the UK in 1993 and 2004, respectively.

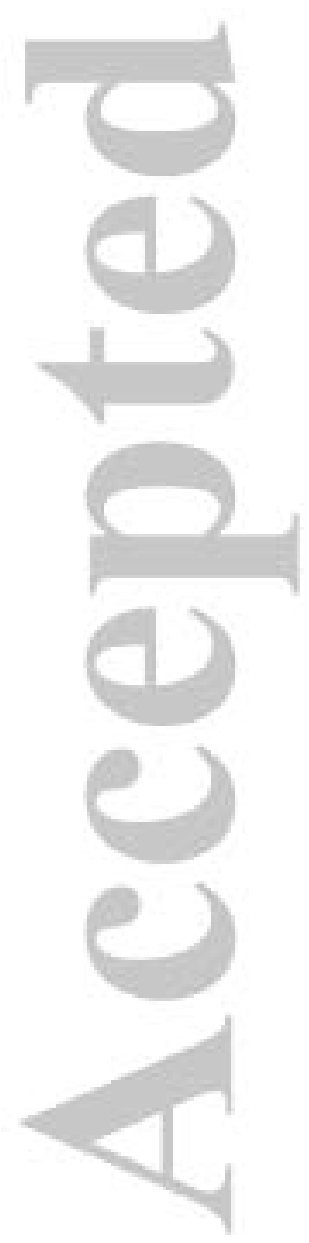




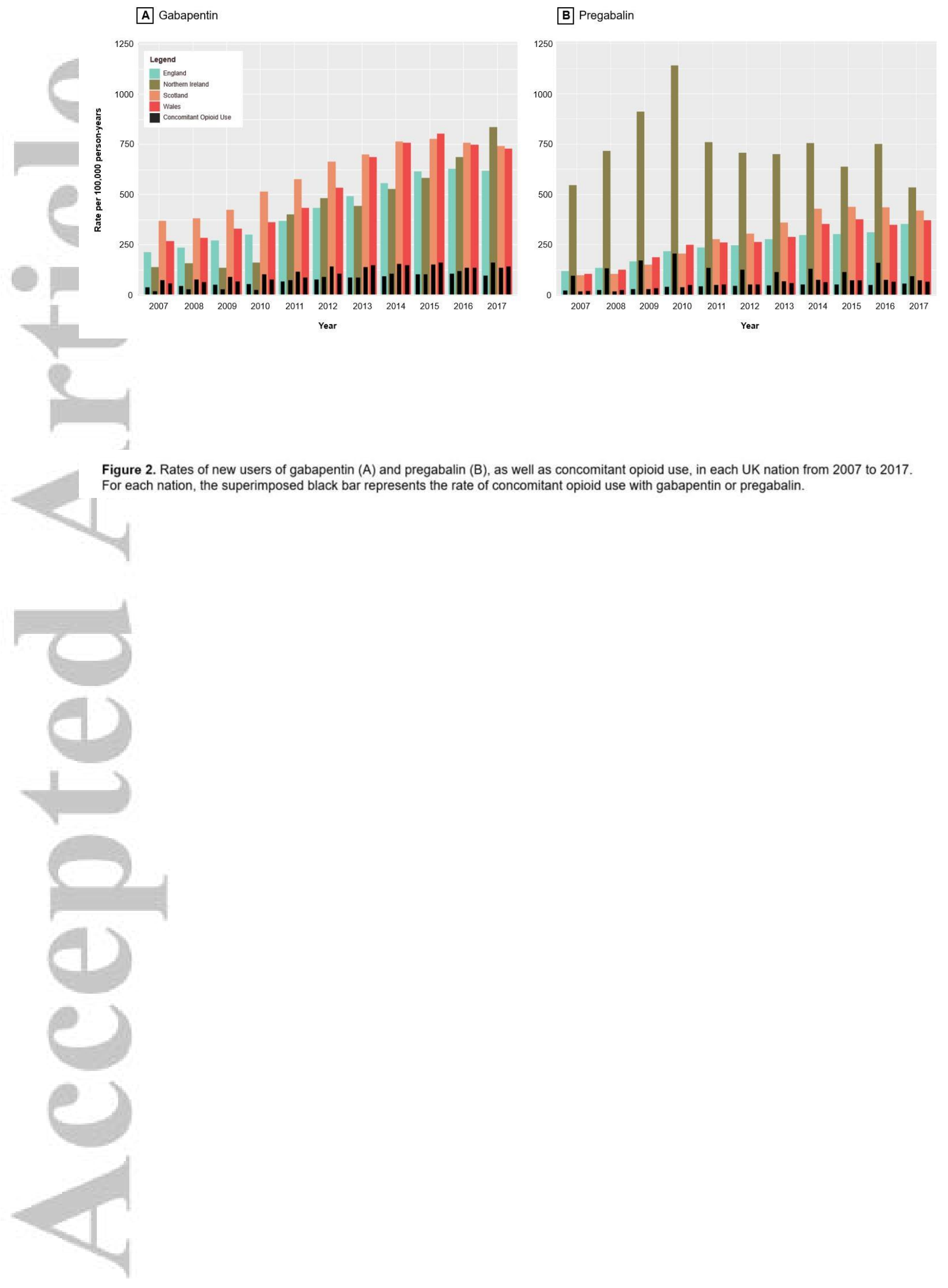

